\title{
Experimental and computational study of light scattering by irregular particles with extreme refractive indices: hematite and rutile
}

\author{
O. Muñoz ${ }^{1}$, H. Volten ${ }^{2}$, J. W. Hovenier ${ }^{2}$, M. Min², Y. G. Shkuratov ${ }^{3}$, J. P. Jalava ${ }^{4}$, \\ W. J. van der Zande ${ }^{5}$, and L. B. F. M. Waters ${ }^{2,6}$ \\ 1 Instituto de Astrofísica de Andalucía, CSIC, Camino Bajo de Huétor 50, Granada 18008, Spain \\ e-mail: olga@iaa.es \\ 2 Astronomical Institute "Anton Pannekoek", University of Amsterdam, Kruislaan 403, 1098 SJ Amsterdam, The Netherlands \\ 3 Institute of Astronomy, Kharkov National University, 35 Sumskaya St., Kharkov 61022, Ukraine \\ 4 Kemira Pigments Oy, Titaanitie 1, 28840 Pori, Finland \\ 5 Institute for Molecules and Materials, Radboud University Nijmegen, PO Box 9010, 6500 GL Nijmegen, The Netherlands \\ ${ }^{6}$ Instituut voor Sterrenkunde, Katholieke Universiteit Leuven, Celestijnenlaan 200B, 3001 Heverlee, Belgium
}

Received 29 June 2005 / Accepted 1 August 2005

\section{ABSTRACT}

We present measurements of the complete scattering matrix as a function of the scattering angle of randomly oriented irregular hematite and rutile particles. The measurements were made at a wavelength of $632.8 \mathrm{~nm}$ in the scattering angle range from 5-174 degrees. Apart from their astronomical interest, these two samples are extremely interesting from a theoretical point of view, because they both have high real parts of the refractive index (about 3.0 for the hematite and 2.73 for the rutile). In addition, the hematite sample has a high imaginary part of the refractive index $k$, with values between $10^{-1}$ and $10^{-2}$, whereas rutile is a non-absorbing material $(k \approx 0)$ at the studied wavelength. The scattering patterns of these mineral particles are quite similar to each other but show remarkable differences when compared to the results obtained for irregular mineral particles with moderate real parts of the refractive index. The measured results for both samples were compared with results of Mie calculations for projected surface equivalent spheres and T-matrix calculations for various spheroidal and cylindrical shapes. Both the experimental and theoretical results presented in this work seem to indicate that the scattering behavior of irregular mineral particles that have a high real part of the refractive index is not very dependent on the shape of the particles. In this case, Mie theory may give reasonable results despite the irregular shapes of the particles.

Key words. solar system: general - scattering - polarization - dust, extinction

\section{Introduction}

Hematite is an iron oxide, $\mathrm{Fe}_{2} \mathrm{O}_{3}$, that is believed to be the main material that gives Mars its red color (Banin et al. 1993; Morris \& Lauer 1990). The study of hematite material is currently an astronomical hot topic since recent investigations have revealed that the existence of hematite $\left(\mathrm{Fe}_{2} \mathrm{O}_{3}\right)$ concretions in Mars could imply a water-rich past in the shallow subsurface on Mars (Chan et al. 2004). Moreover, hematite is one of the mineral aerosol components that can be found in the Earth's atmosphere (Sokolik et al. 1999). Rutile, $\mathrm{TiO}_{2}$, dust particles are thought to be the most likely nucleation seeds in oxygenrich circumstellar shells (Jeon et al. 1999, 2003). In addition, rutile is an accessory component of the lunar regolith (Frondel 1975).

The scattering properties of these kinds of irregular dust particles are an important diagnostic tool for the remote detection and the retrieval of information on the physical properties of the grains. Although in recent years tremendous progress has been made in computing light scattering properties of irregular mineral particles (see e.g. Mishchenko et al. 2000a, 2002; Wriedt 2002; Nousiainen et al. 2003), these methods are still not completely adequate to simulate light scattering by realistic shapes. Scattering properties of spherical particles can be calculated with the Mie theory (Mie 1908) without any constraints concerning sizes or refractive indices of the particles. However, many laboratory (see e.g. West et al. 1997; Muñoz et al. 2000, 2001, 2004; Volten et al. 2001) and computational results (see e.g. Mishchenko et al. 1997, 2000a, 2002; Krotkov et al. 1999) show that light-scattering properties of irregular mineral particles can differ significantly from those of volume equivalent spheres with the same refractive index. Still, a large amount of dust retrievals are based on the Mie theory for spherical 
particles. Therefore, there is a lot of interest in studying under which circumstances this theory is adequate for computing the scattering behavior of non-spherical particles.

In this paper, we present measurements of the complete scattering matrix as a function of the scattering angle of two samples of randomly oriented irregular mineral dust particles: hematite $\left(\mathrm{Fe}_{2} \mathrm{O}_{3}\right)$, and rutile $\left(\mathrm{TiO}_{2}\right)$. The measurements were performed at $632.8 \mathrm{~nm}$. The experimentally determined scattering matrix elements of a rutile sample in water have already been published by Volten et al. (1999). In this paper we present measurements of a similar sample of rutile particles dispersed in air. Apart from their astronomical interest, the two samples considered in this paper are very interesting from a theoretical point of view because they present quite extreme real parts, $n$, of the refractive index at $632.8 \mathrm{~nm}: \sim 3.0$ for the hematite sample, and 2.73 for the rutile sample. Moreover, hematite is highly absorbing with an imaginary part of the refractive index, $k$, between $10^{-2}$ and $10^{-1}$ at $632.8 \mathrm{~nm}$, whereas rutile is a non-absorbing material in the visible $(k \approx 0)$. Indeed, rutile pigments are widely employed in industry for their extreme whiteness. Thus, we have experimentally studied the scattering behavior of two samples with high real parts of the refractive index but with very different absorption properties. The measured results for hematite and rutile particles were compared to the experimentally determined average scattering matrix for irregular mineral silicate particles obtained by Volten et al. (2001). This matrix was obtained by measuring at two wavelengths (441.6 and $632.8 \mathrm{~nm}$ ) the scattering matrices as functions of the scattering angle of seven different mineral samples, all of them consisting of irregularly shaped particles with moderate refractive indices i.e., the real part is between 1.5 and 1.7 and the imaginary part between 0 and $10^{-3}$, but with different size distributions.

T-matrix calculations of the phase function (Mishchenko et al. 1997, 2002) and degree of linear polarization for incident unpolarized light (Mishchenko et al. 1994, 2002) of polydisperse prolate and oblate spheroids suggest that, by increasing the imaginary part of the refractive index, the differences in the scattering behavior between spherical and nonspherical particles vanish. These authors performed calculations for polydisperse spheroids with moderate real parts of the refractive index (1.5, and 1.53) varying the imaginary part of the refractive index within a wide range of values. In this work we have considered higher values of the real part of the refractive index and experimental and theoretical results. Our experimental results were compared to results of Mie calculations for spheres and T-matrix calculations for spheroids and cylinders with various values of the real and imaginary parts of the refractive index. In this way we studied more systematically under which circumstances the differences in the scattering behavior between spherical and non-spherical particles become small, i.e. under which circumstances the Mie theory for spherical particles can be used as a good approximation for simulating light scattering by non-spherical particles.

In Sect. 2, we give a brief description of the scattering setup used to perform the light scattering measurements. In Sect. 3 we present some physical properties of the samples used in this work. The measurements are presented in Sect. 4. In Sect. 5 the measurements are them compared with results of Mie calculations for spheres and T-matrix calculations for spheroids, and cylinders. In Sect. 6 we present the conclusions of this work.

\section{Scattering setup}

A detailed description of the experimental setup used to measure the scattering matrices is given by Hovenier (2000), and Hovenier et al. (2003). We give a brief summary here. We used a HeNe laser $(632.8 \mathrm{~nm}, 5 \mathrm{~mW})$ as a light source. The laser light passes through a polarizer and an electro-optic modulator. The modulated light is subsequently scattered by an ensemble of randomly oriented particles located in a jet stream produced by an aerosol generator. The scattered light passes through a quarter-wave plate and an analyzer (both optional) and is detected by a photomultiplier tube which moves in steps along a ring. A range in scattering angles is covered from approximately $5^{\circ}$ (nearly forward scattering) to about $174^{\circ}$ (nearly backward scattering). Another photomultiplier is used to monitor the flux of scattered light at a fixed position. The signal of the monitor is used to correct for possible fluctuations in the scattered signal. We employ polarization modulation in combination with lock-in detection to determine all elements $F_{i j}$, of the $4 \times 4$ scattering matrix $\mathbf{F}$ of the ensemble of particles as a function of the scattering angle $\theta$. Here it is assumed that the particles are much farther apart than their linear dimensions and that multiple scattering by the particles can be neglected. The elements of the scattering matrix depend on the number and physical properties of the particles, the wavelength of the incident radiation, and the direction of the scattered light, which for randomly oriented particles, is sufficiently described by means of the scattering angle $(\theta)$. We normalize all measured phase functions to 1 at $30^{\circ}$. For convenience, all matrix elements (except $F_{11}$ itself) are normalized to $F_{11}$; that is, we consider $F_{i j} / F_{11}$, with $i, j=1$ to 4 except for $i=j=1$. Due to reciprocity, $F_{21}(\theta) / F_{11}(\theta)=F_{12}(\theta) / F_{11}(\theta)$ and $F_{43}(\theta) / F_{11}(\theta)=-F_{34}(\theta) / F_{11}(\theta)$, and all other off-diagonal elements divided by $F_{11}$, vanish identically within experimental errors. Thus $-F_{12}(\theta) / F_{11}(\theta)$ is the degree of linear polarization for incident unpolarized light. For all measurements reported in this paper, we have investigated the reliability of the measured angular distributions by checking that all satisfy the Cloude test (Hovenier \& van der Mee 2000) within the experimental errors.

\section{Samples}

Hematite is a ferric oxide mineral $\left(\mathrm{Fe}_{2} \mathrm{O}_{3}\right)$ that may be formed by a variety of processes that often involve the presence of water. It is believed to be an important component of martian dust and in addition, it is an important constituent of terrestrial aerosols (Sokolik \& Toon 1999; Höller et al. 2003). Hematite is a birefringent material with a large real and imaginary part of the refractive index in the visible part of the spectrum. The real part of the refractive index, $n$ at $632.8 \mathrm{~nm}$, has a value 2.9 for the extraordinary and 3.1 for the ordinary axis (Sokolik \& Toon 1999). In our calculations we assumed an average value 


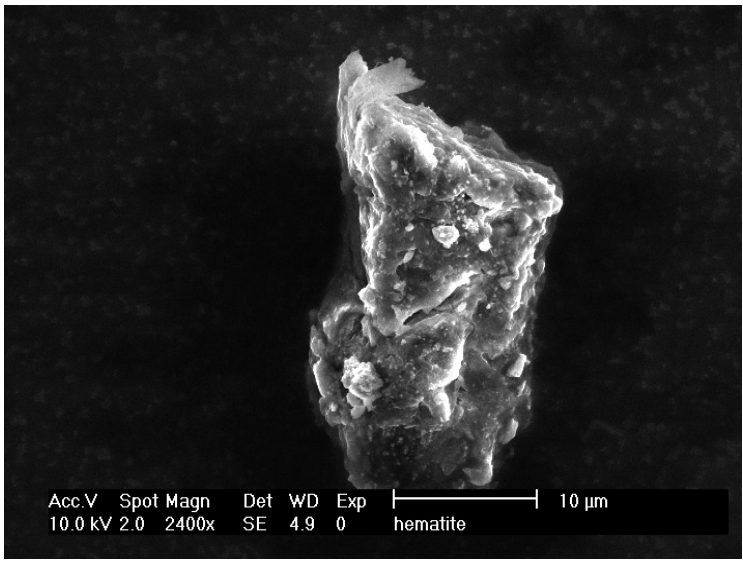

Fig. 1. Scanning Electron Microscope (SEM) image of a hematite particle.

of 3.0 for the real part of the refractive index. The imaginary part, $k$, ranges between $10^{-2}$ and $10^{-1}$ (Sokolik \& Toon 1999).

The normalized projected-surface-area distribution $S(\log r)$ of our hematite sample was measured by a Fritsch laser particle sizer that employs a diffraction method without making assumptions about the refractive indices of the particles (Konert $\&$ Vandenberghe 1997). Here, $S(\log r) \mathrm{d} \log r$ is the relative contribution by projected-surface-equivalent spheres with radii in the size range $\log r$ to $\log r+\mathrm{d} \log r$ to the total projected surface per unit volume of space. We define $r$ as the radius of a sphere, called equivalent sphere, having the same average (over all directions) projected surface area. From the measured projected-surface area distribution, we obtained the values of the effective radius, $r_{\text {eff }}$, and effective variance, $v_{\text {eff }}$, defined as follows:

$r_{\text {eff }}=\frac{\int_{0}^{\infty} r \pi r^{2} n(r) \mathrm{d} r}{\int_{0}^{\infty} \pi r^{2} n(r) \mathrm{d} r}$,

$v_{\text {eff }}=\frac{\int_{0}^{\infty}\left(r-r_{\text {eff }}\right)^{2} \pi r^{2} n(r) \mathrm{d} r}{r_{\text {eff }}^{2} \int_{0}^{\infty} \pi r^{2} n(r) \mathrm{d} r}$,

where $n(r) \mathrm{d} r$ is the fraction of the total number of projected surface equivalent spheres with radii in the size range $[r, r+\mathrm{d} r]$ per unit volume of space (Hansen \& Travis 1974). Here, $n(r)$ is computed from $S(\log r)$ with $n(r)=N(\log r) / r \ln 10$, where $N(\log r)$ is defined so that $N(\log r) \mathrm{d}(\log r)$ is the relative number of equivalent spheres with radii in the interval $\mathrm{d}(\log r)$. For more detailed information on the size distribution, we refer to Volten et al. (2005). Our hematite sample has an $r_{\text {eff }}$ of $0.4 \mu \mathrm{m}$ and a $v_{\text {eff }}$ of 0.6. In Fig. 1, we present a Scanning Electronic Microscope (SEM) picture of a hematite particle as an example of the shape of this type of particles. In that picture we can see that the hematite particles exhibit very irregular shapes with a layered structure.

The rutile $\left(\mathrm{TiO}_{2}\right)$ studied in this work is a commercial sample provided by Kemira Pigments Oy, Pori, Finland. Rutile is a birefringent material with a real part of the refractive index, $n$, at $632.8 \mathrm{~nm}$ of 2.87 for the extraordinary and 2.58 for the ordinary axis. In our calculations we assumed an average value of 2.73 for the real part of the refractive index. In contrast to

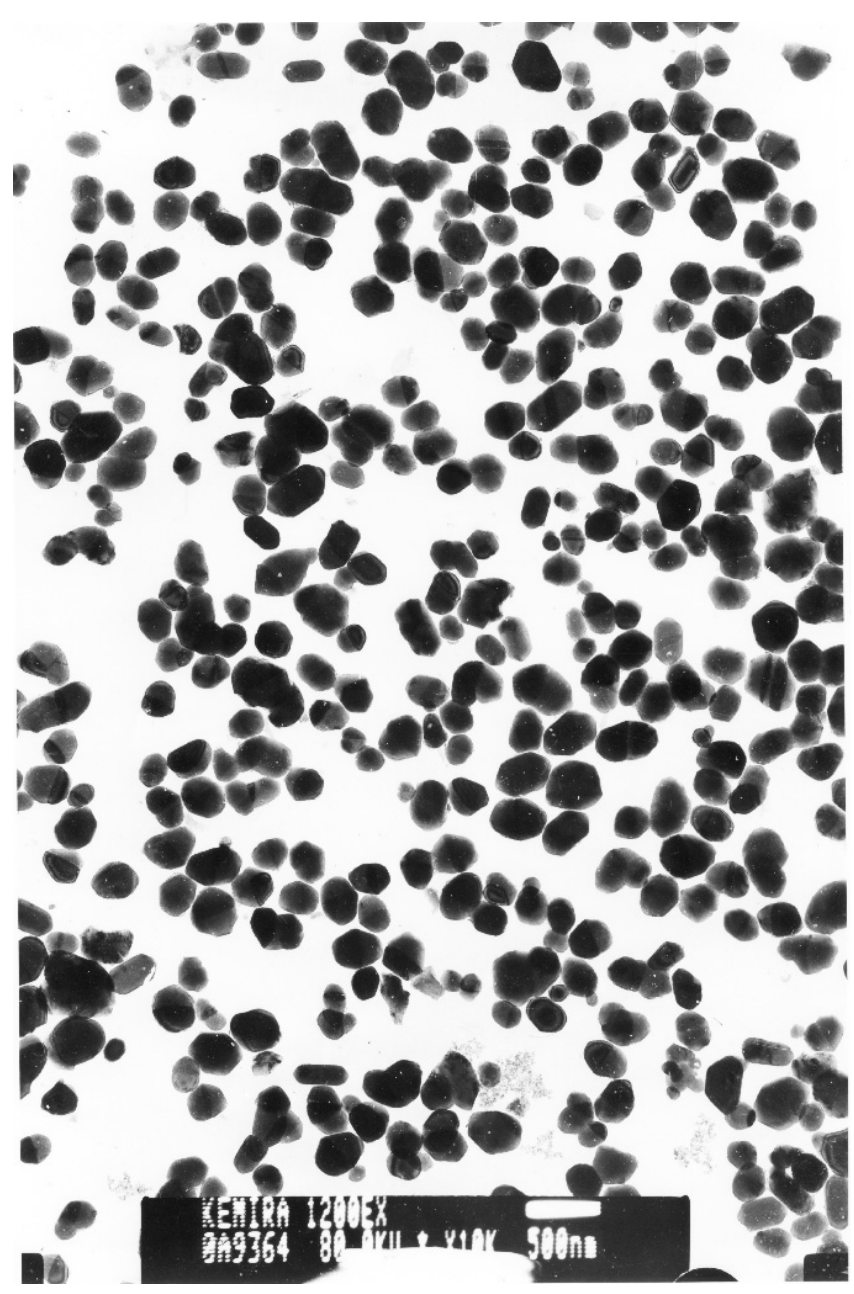

Fig. 2. Transmission Electron Microscope (TEM) image of the rutile sample.

the hematite sample, the imaginary part of the refractive index, $k$, of rutile is zero. In Fig. 2 we show a Transmission Electron Microscope (TEM) picture of the rutile sample. The rutile crystals have two axes of the same length, whereas the third axis is generally the longest. Therefore, most rutile particles tend to have a prolate shape. Using a turbidity spectrum method (Jalava et al. 1998, 2000), a size distribution was determined with a mean radius of $117 \mathrm{~nm}$ and a standard deviation of $28 \mathrm{~nm}$ expressed in terms of volume equivalent spheres. This corresponds to an $r_{\text {eff }}$ of $0.13 \mu \mathrm{m}$ and a $v_{\text {eff }}$ of 0.5 . The length/width distribution has a length/width of 1.42 and a standard deviation of 0.24 ; the mean width is $207 \mathrm{~nm}$ with a standard deviation of $48 \mathrm{~nm}$.

\section{Measurements}

In Figs. 3 and 4 we show the measured scattering matrix elements as functions of the scattering angle for the hematite and rutile samples at $632.8 \mathrm{~nm}$. The scattering functions or phase functions, $F_{11}(\theta)$, are shown on a logarithmic scale and are normalized to 1 at $30^{\circ}$. The experimental errors are indicated by error bars. When no error bar is shown, the value of the standard deviation of the mean value is smaller than 

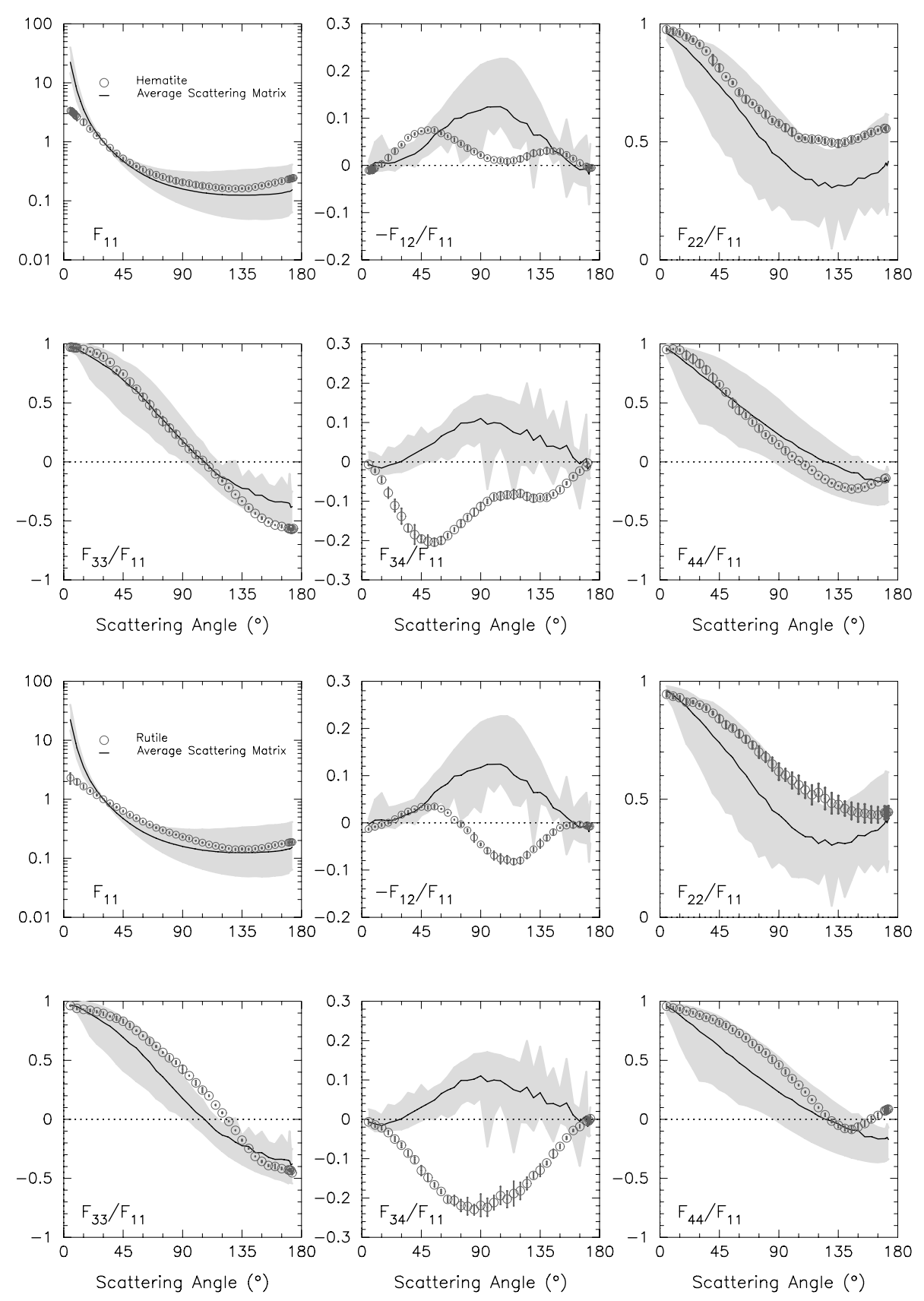

Fig. 3. Measured scattering matrix as function of the scattering angle for hematite particles at $632.8 \mathrm{~nm}$ (open circles). The measurements are presented together with their error bars. In case no error bars are shown, they are smaller than the symbols. Solid lines correspond to the average scattering matrix for mineral particles. This average was obtained from seven samples of irregularly shaped mineral particles at 441.6 and $632.8 \mathrm{~nm}$. The domains occupied by the aerosol measurements are indicated in gray (Volten et al. 2001).

Fig. 4. Same as Fig. 3 but for the rutile sample. the plotted symbol. Despite the large difference in the imaginary part of the refractive index, the scattering behaviors of the hematite and rutile samples are not very dissimilar. In contrast, the two samples show quite different scattering patterns when compared to the typical scattering behavior of irregular mineral particles with moderate real parts of the refractive index (see e.g. Muñoz et al. 2000, 2001, 2004; Mishchenko et al. 2000b; Volten et al. 2001). To illustrate this in Figs. 3 and 4 we show the measured scattering matrix elements for hematite and rutile together with the experimentally determined average scattering matrix for irregular mineral particles (Volten et al. 2001). This average was obtained from seven samples of irregularly shaped mineral particles: feldspar, red clay, quartz, Pinatubo volcanic ash, loess, Lokon volcanic ash, and Sahara sand at two wavelengths, namely 441.6 and $632.8 \mathrm{~nm}$. The average scattering matrix is displayed in Figs. 3 and 4 by a solid line. The area between the highest and lowest measured values are indicated in gray. The samples used to obtain the average scattering matrix represent a broad range in size distributions with effective radii varying between 1.0 and 8.2 micron. Moreover, all samples used to obtain the average scattering matrix have moderate real parts of the refractive index $(1.5 \leq n \leq 1.7)$. The imaginary parts of the refractive index range between 0 and $10^{-3}$.

In Figs. 3 and 4 we can see that the degree of linear polarization for incident unpolarized light as a function of the scattering angle (i.e. $-F_{12}(\theta) / F_{11}(\theta)$ ) of the hematite and rutile samples differs considerably from the average curve. In both cases it shows a rather low double maximum around 50 and 140 degrees. In contrast, the average degree of linear polarization shows a characteristic bell shape with a maximum around 90 degrees and a negative branch at large scattering angles. 

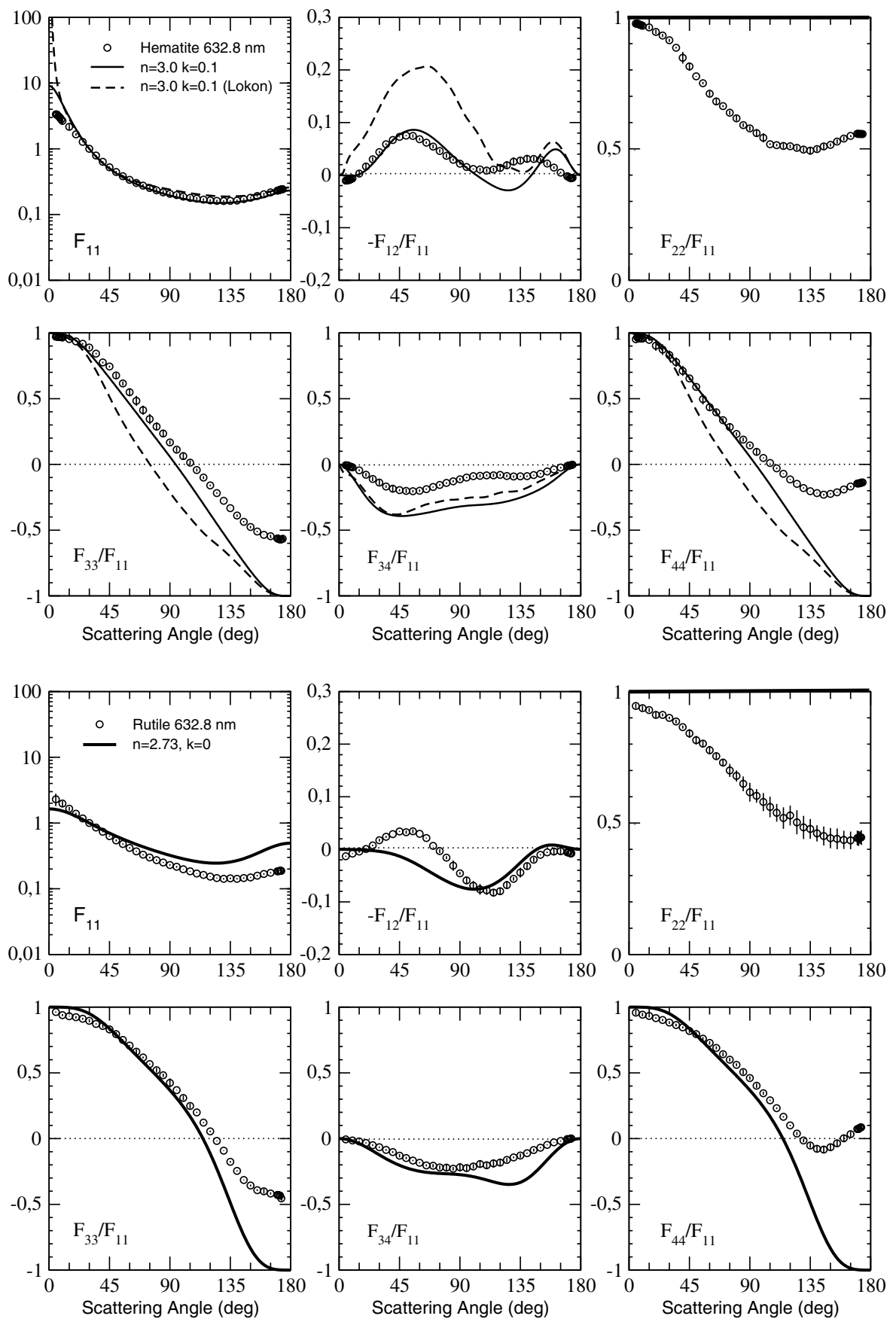

Fig. 5. Measured scattering matrix elements as functions of the scattering angle at $632.8 \mathrm{~nm}$ for the hematite sample (open circles). The measurements are presented together with results of Mie calculations for spheres with the same number distribution, $n(r)$, and refractive index as the hematite sample (solid lines). Dashed lines correspond to Mie calculations for the size distribution of the Lokon sample presented by Volten et al. (2001), but with the refractive index of the hematite sample $(n=3$ and $k=0.1$ ).

Fig. 6. Measured scattering matrix elements as functions of the scattering angle at $632.8 \mathrm{~nm}$ for the rutile sample (open circles). The measurements are presented together with results of Mie calculations for a gamma distribution of spheres with the same $r_{\text {eff }}, v_{\text {eff }}$, and refractive index as the rutile sample has.
Moreover, the measured $F_{34}(\theta) / F_{11}(\theta)$ for the hematite and rutile samples is found to differ appreciably from the average curve for irregular mineral particles with moderate refractive indices. This ratio has negative values at all measured scattering angles for the hematite and rutile samples, whereas it is positive at almost all scattering angles for the average $F_{34}(\theta) / F_{11}(\theta)$. In addition, the measurements for the hematite sample show a bimodal function with a primary minimum around 60 degrees and a secondary minimum around 160 degrees.

Differences between the ratios $F_{33}(\theta) / F_{11}(\theta)$ and $F_{44}(\theta) / F_{11}(\theta)$ for hematite and rutile particles compared to irregular mineral particles are not so spectacular although they also lie out of the domains of the average curve at some measured scattering angles. The measured $F_{22}(\theta) / F_{11}(\theta)$ for the hematite sample shows the typical behavior for irregular mineral particles decreasing from almost 1 at angles close to the forward direction to a minimum and then increasing again at back-scattering angles. However, this ratio stays out of the domain of the average curve at almost all measured scattering angles. The $F_{22}(\theta) / F_{11}(\theta)$ for the rutile sample does not show the increase at back-scattering angles.

The hematite and rutile scattering functions or phase functions, $F_{11}(\theta)$, range over little more than one order of magnitude for the measured angles. In contrast, the average scattering function ranges over more than two orders of magnitude for the angles covered in the measurements, being strongly peaked toward smaller angles. These differences in the forward scattering peaks are probably due to the differences in sizes between 
our hematite and rutile samples (see Sect. 3) and the dust samples used to construct the average scattering matrix, since forward scattering peaks depend strongly on the sizes of the particles (Mishchenko et al. 1997, 2000a).

Apart from the differences in the forward scattering peaks, we do not think the differences in size between the hematite and rutile samples, on the one hand, and the samples used to obtain the average scattering matrix, on the other, are strong enough to account for the significant differences observed in almost all scattering matrix elements as functions of the scattering angle. This assumption is based on previous experimental studies (Volten et al. 2001; Muñoz et al. 2001) and is further checked in the next section. As mentioned, all samples used to obtain the average scattering matrix have moderate real parts of the refractive index with values ranging between 1.5 and 1.7. In contrast, the hematite and rutile samples have very high values of the real part of their refractive indices (about 3.0 and 2.73 , respectively). To this we attribute the main differences in scattering behavior of the hematite and the rutile sample when compared with the average scattering matrix.

\section{Calculations and discussion}

In Figs. 5 and 6 we compare the measured scattering matrix elements as functions of the scattering angle for the hematite and rutile samples with results of Mie calculations for homogeneous optically nonactive spherical particles. For the calculations presented in Fig. 5 we employed the refractive index and measured number distribution, $n(r)$, of the hematite sample. According to Jalava et al. (1998), the gamma distribution is a suitable distribution for describing both the width and the length/width distributions of the rutile particles. Thus, for the Mie calculations of the rutile sample, we assumed a gamma distribution with the same $r_{\text {eff }}$ and $v_{\text {eff }}$ as measured. The calculated phase functions were also normalized to 1 at $30^{\circ}$. As shown in Fig. 5, the agreement between measured and calculated values for the hematite sample is surprisingly good for some elements of the scattering matrix despite the high irregularity in shapes of the hematite particles (see Fig. 1). In particular, the shape of the phase function shows an almost perfect fit except at forward scattering angles. The degree of linear polarization for unpolarized incident light as function of the scattering angle also shows a remarkably good fit reproducing the measured low double maximum. The calculated $F_{34}(\theta) / F_{11}(\theta)$ shows a qualitative agreement with the measurements. It also presents negative values at all scattering angles with a principal minimum around 30 degrees. The main discrepancies are related to the $F_{22}(\theta) / F_{11}(\theta)$ ratio, since for spherical particles this is equal to unity at all scattering angles, whereas in our measurements it deviates considerably from 1 with a minimum of 0.5 at 135 degrees.

Like for the hematite sample, the scattering matrix elements as functions of the scattering angle of the rutile sample (Fig. 6) agrees qualitatively well between measured and calculated values of some elements of the scattering matrix. The calculated values of $F_{11}(\theta)$ are significantly larger than the measured values at scattering angles larger than about 50 degrees. However, a moderatly good fit is obtained at the forward scattering peak. The calculated $-F_{12}(\theta) / F_{11}(\theta)$ reproduces the measured one quite well, in particular at scattering angles larger than about 100 degrees. The measured values for $F_{34}(\theta) / F_{11}(\theta)$ show an inverted bell shape, whereas the calculated values $F_{34}(\theta) / F_{11}(\theta)$ present a bimodal shape with a principal minimum at around 125 degrees. Still, measured and calculated values for $F_{34}(\theta) / F_{11}(\theta)$ present negative values at all scattering angles showing a reasonable good fit at scattering angles between 5 and around 90 degrees. As in the case of the hematite sample, the main discrepancies are related to the $F_{22}(\theta) / F_{11}(\theta)$.

As mentioned in the previous section, the characteristic scattering behavior of the hematite and rutile samples when compared with the average scattering matrix for silicate particles, seems to be related to the high real part of the refractive index of its particles. However, the particles of these samples are smaller than the particles of the samples used to obtain the average scattering matrix. In order to check whether this difference in sizes could be producing the differences in the scattering behavior, we repeated the Mie calculations by assuming the size distribution of Lokon volcanic ash but fixing the refractive index of the hematite sample (Fig. 5, dashed line). Lokon volcanic ash is one of the samples used by Volten et al. (2001) to construct the average scattering matrix. The Lokon sample has a relatively large $r_{\text {eff }}(7.1 \mu \mathrm{m})$ and $v_{\text {eff }}$ (2.56). Although there are differences with the calculated results for the measured size distribution of the hematite particles, we still qualitatively reproduce the scattering pattern of the hematite sample. Therefore, the sizes of the hematite and rutile particles do not seem to be an important factor in causing the differences with the average scattering matrix.

The reasonable fit obtained for the hematite sample and the rutile sample by assuming spherical particles seems to indicate that, for irregular particles with a high real part of the refractive index, Mie computations may be a relatively good approach. Although the particles cannot be considered to be large, the experimentally measured scattering behavior of the samples seems to be in line with a general theorem formulated by van de Hulst (1957; Sect. 8:42): "The scattering pattern caused by reflection on very large convex particles with random orientation is identical with the scattering pattern by reflection on a very large sphere of the same material and surface conditions". For such very large convex particles, rays hitting the surface of a particle are partially reflected and partially refracted. The energy that does not emerge is lost by absorption inside the particle. For very large particles, the diffracted light can be distinguished from the remaining scattering pattern. For very large particles with large real and imaginary parts of the refractive index, we have only reflected and diffracted light since the refracted part is absorbed within the particle. According to the results presented in Figs. 5 and 6, it seems like the reasoning of van de Hulst still holds to a certain extent for particles with the size distributions of our samples if they have a large real part of the refractive index. A high imaginary part of the refractive index should increase the effect, although this is not visible in Figs. 5 and 6. For particles with high imaginary parts of the refractive index and values of $n$ smaller than that of rutile and hematite, such an effect is 

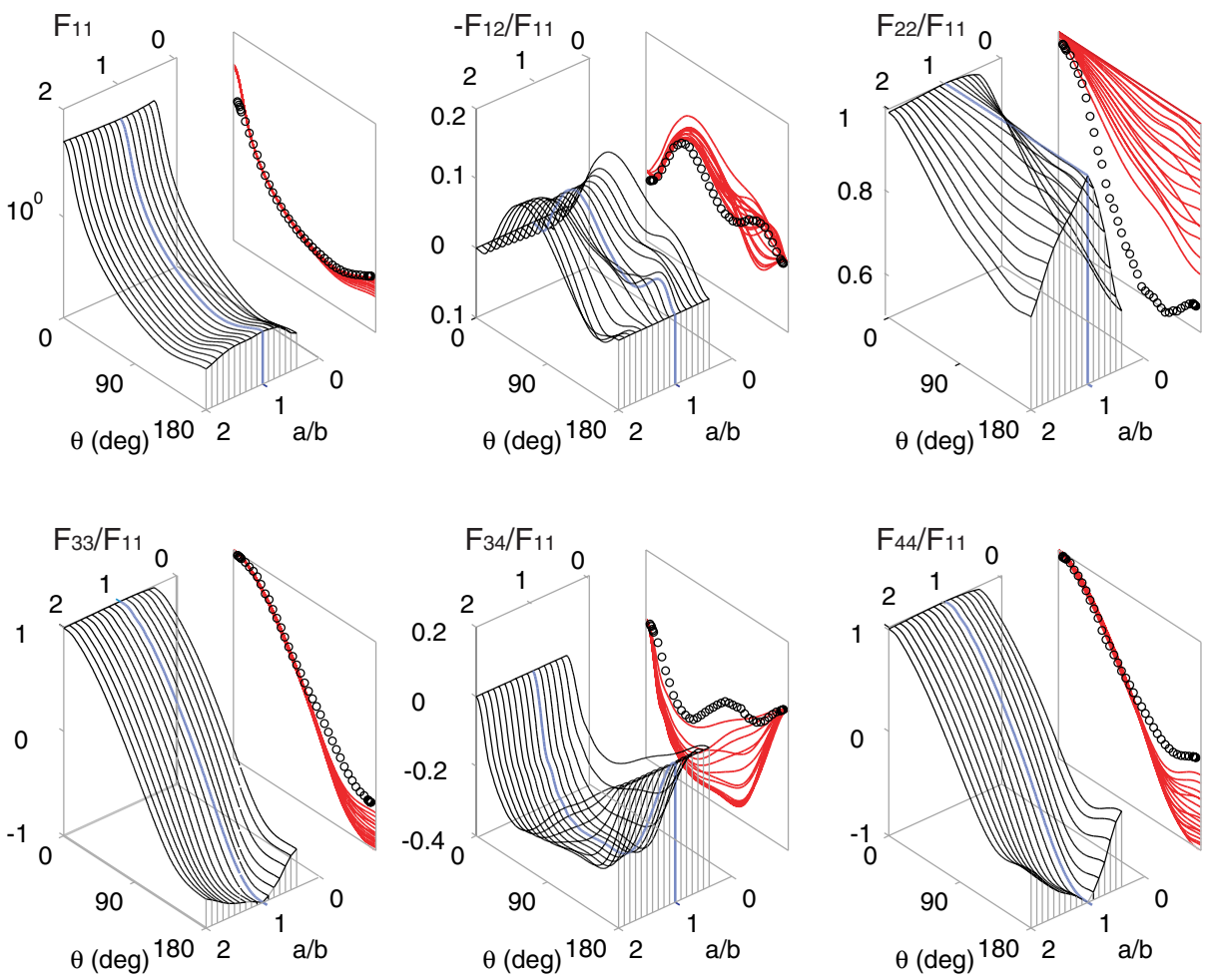

Fig. 7. Calculated elements of the scattering matrix for randomly oriented spheroids versus scattering angle and aspect ratios $(\mathrm{a} / \mathrm{b})$. For these calculations we used the same refractive index and a power law distribution with the same $r_{\text {eff }}$ and $v_{\text {eff }}$ as for our hematite sample. Blue lines correspond to calculations for $\mathrm{a} / \mathrm{b}$ equal to 1 . In the back panels we present the projections of the calculated scattering matrix elements (solid red lines) as functions of the scattering angle together with the measured scattering matrix elements for the hematite sample (circles).
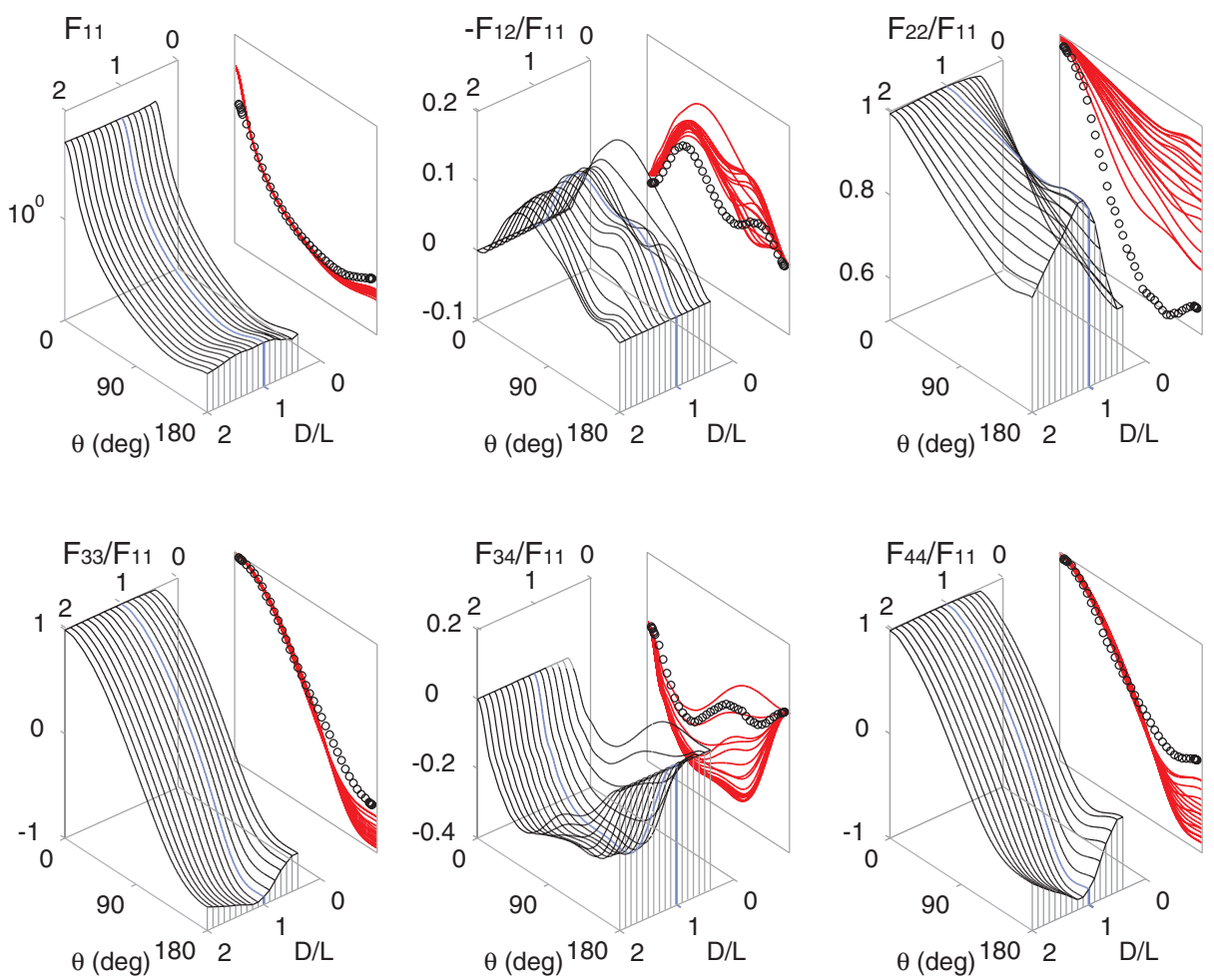

Fig. 8. Same as Fig. 7 but for cylinders whose diameter-to-length ratio are defined by $\mathrm{D} / \mathrm{L}$.

clearly noticeable as shown by the T-matrix calculations for spheroidal particles presented by Mishchenko \& Travis (1994) and Mishchenko et al. (1997, 2002). They give some examples for relatively small particles that illustrate the general theorem proposed by van de Hulst (1957). Mishchenko \& Travis (1994) studied the effect of the imaginary part of the refractive index on the degree of linear polarization as a function of the scattering angle of different polydisperse samples of spheroids in random orientation. These authors fixed the real part of the refractive index to 1.5 , varying the imaginary part between 0.003 and 0.3 . They observed that by increasing the imaginary part of the refractive index for a fixed $n$, the differences between calculated values for $-F_{12}(\theta) / F_{11}(\theta)$ for spherical and nonspherical particles vanish. Moreover, Mishchenko et al. (1997) 
used the T-matrix method to compute the phase function of polydisperse randomly-oriented spheroids with the real part of the refractive index fixed to 1.53 and the imaginary part ranging between 0.0005 and 0.5 . They proved that nonsphericalspherical differences decrease as the imaginary part of the refractive index increases for values of $n$ of about 1.5.

In order to further investigate the shape effects for scattering by randomly oriented convex particles with high real parts of the refractive index, we performed calculations by using the extended precision T-matrix algorithm developed by Mishchenko et al. (1996) (http://www.giss.nasa.gov/ $\sim$ crmim/t_matrix.html). In these calculations we chose a power law distribution to represent the number distribution, $n(r)$, of our hematite sample. Here, the particle size is also defined in terms of equal averaged projected area. The exponent of the power law distribution assumed in the T-matrix code is equal to -3 . This value is quite close to the exponent of the power law distribution that best fits the $n(r)$ of the hematite sample (-2.65). In these calculations we assumed the same $r_{\text {eff }}$ and $v_{\text {eff }}$ and the same refractive index as the hematite sample has. The minimal and the maximal radii, $r_{\min }$ and $r_{\max }$, of the size distribution are calculated from the given $r_{\text {eff }}$ and $v_{\text {eff }}$. The calculated values for $r_{\min }$ and $r_{\max }$ are consistent with the minimal and maximal radii of the hematite sample. Therefore, we assumed the power law distribution with exponent -3 as a good approximation for the number distribution of our hematite sample.

The calculations were carried out for prolate and oblate spheroids with various ratios of the horizontal to the vertical (rotational) axes, $\mathrm{a} / \mathrm{b}$, i.e. for aspect ratios ranging from 0.4 to 2.0 (Fig. 7). All calculated phase functions presented in this section have been normalized to 1 at $30^{\circ}$. The calculated values are presented together with the experimentally determined scattering matrix for the hematite sample. As in the case of the Mie calculations for the hematite sample, the calculated values show a qualitative agreement with the measurements. The main discrepancies are related again to the $F_{22}(\theta) / F_{11}(\theta)$ ratio. In addition, the calculated values for different aspect ratios are not so very dissimilar. We repeated the calculations by assuming cylindrical shapes with different ratios of the diameter to the length $(\mathrm{D} / \mathrm{L})$. The results are presented in Fig. 8. The calculated results are again very similar to the values obtained for spherical particles and the measured scattering matrix for the hematite particles, in spite of the big differences in the shapes of the particles.

We repeated the T-matrix calculations for the rutile particles (Figs. 9 and 10). Due to computational constraints, we could not use a gamma distribution to represent the size distribution of the rutile sample. Instead we assumed a standard power law distribution with the same effective radius and variance and the same refractive index as the rutile sample has. In this case the size distribution is specified in terms of volume equivalent spheres. As shown in Figs. 9 and 10, the calculated scattering matrix elements as functions of the scattering angle seem to be more sensitive to the shape of the particles than in the case of the hematite sample.

We studied the effect of different values of the real and imaginary parts of the refractive index on the scattering pattern in more detail. We then repeated the calculations for the following combinations of real and imaginary parts of the refractive index:

$n=1.5 ; k=0,0.001,0.01,0.1$

$n=4 ; k=0,0.001,0.01,0.1$.

We assumed a power law distribution with the same $r_{\text {eff }}$ and $v_{\text {eff }}$ as the hematite sample, varying the aspect ratio of our spheroids between 0.4 and 1.5 in steps of 0.1 . The calculated results (not shown) corroborate that increasing either $k$ and/or $n$ for any given $n$ helps to decrease nonspherical-spherical differences.

In a broader context, this could be explained as follows. Let us consider the formula of the reflectance, $R$, defined as the ratio of reflected-to-incident irradiance for Fresnel reflection by a flat surface. We first consider normally incident light (Bohren \& Huffman 1983, Sect. 2.7.1). Then,

$R=\frac{(n-1)^{2}+k^{2}}{(n+1)^{2}+k^{2}}$,

where $R$ is the reflectance and $n$ and $k$ are the real and imaginary parts of the refractive index, respectively. In Fig. 11, we present the reflectance, $R$, for normally incident light as a function of $n$ and $k$. Equation (3) and Fig. 11 show, for example, that $R$ takes a value of 0.04 for $n=1.5$ and $k=0$, but increases steeply to about 0.17 if we increase the value of $k$ to 1 , keeping the same value of $n$. If the real part of the refractive index is $n=4$, $R$ is already 0.36 for $k=0$ and increases much less rapidly to 0.38 for $k=1$. Moreover, very high values of $n$ and/or $k$ imply values of reflectance going to 1 , i.e. all incident light is reflected by the flat surface.

Let us now consider oblique incident light. In this case, the reflectance for incident unpolarized light is given by (Bohren \& Huffman 1983, Sect. 2.7.2):

$R=\frac{1}{2}\left(R_{\|}+R_{\perp}\right)$

where $R_{\|}$and $R_{\perp}$ correspond to the reflectances for electric vectors parallel and perpendicular, respectively; to the plane of incidence we have

$R_{\|}=\left|\frac{\cos \Theta_{t}-m \cos \Theta_{i}}{\cos \Theta_{t}+m \cos \Theta_{i}}\right|^{2}$

$R_{\perp}=\left|\frac{\cos \Theta_{i}-m \cos \Theta_{t}}{\cos \Theta_{i}+m \cos \Theta_{t}}\right|^{2}$

where $m$ is the complex refractive index $(m=n+\mathrm{i} k)$, and $\Theta_{i}$ and $\Theta_{t}$ correspond to the angles of incidence and transmission, respectively. $\Theta_{i}$ and $\Theta_{t}$ are related to each other according to the generalized Snell's law:

$\sin \Theta_{t}=\frac{\sin \Theta_{i}}{m}$.

In Fig. 12, we present the reflectance as a function of the angle of incidence, $\Theta_{i}$, for various values of the refractive index. As shown in that figure, the reflectance, $R$, increases with $k$, but 

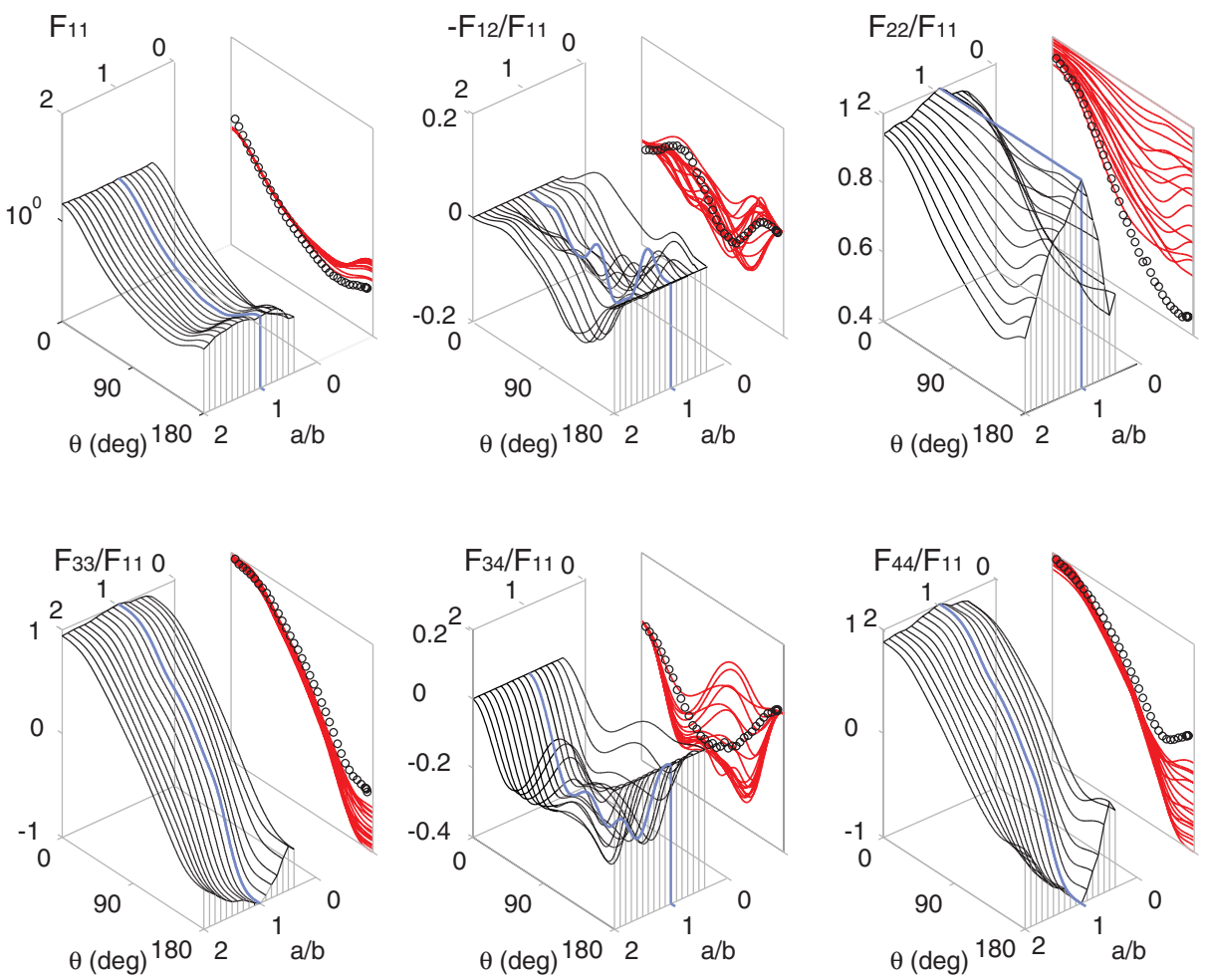

Fig. 9. Calculated elements of the scattering matrix for randomly oriented spheroids versus scattering angle and aspect ratios $(a / b)$. For these calculations we used the same refractive index and a power law distribution with the same $r_{\text {eff }}$, and $v_{\text {eff }}$ as our rutile sample has. Blue lines correspond to calculations with $\mathrm{a} / \mathrm{b}$ equal to 1 . In the back panels, we present the projections of the calculated scattering matrix elements (solid red lines) as functions of the scattering angle together with the measured scattering matrix elements for the rutile sample (circles).
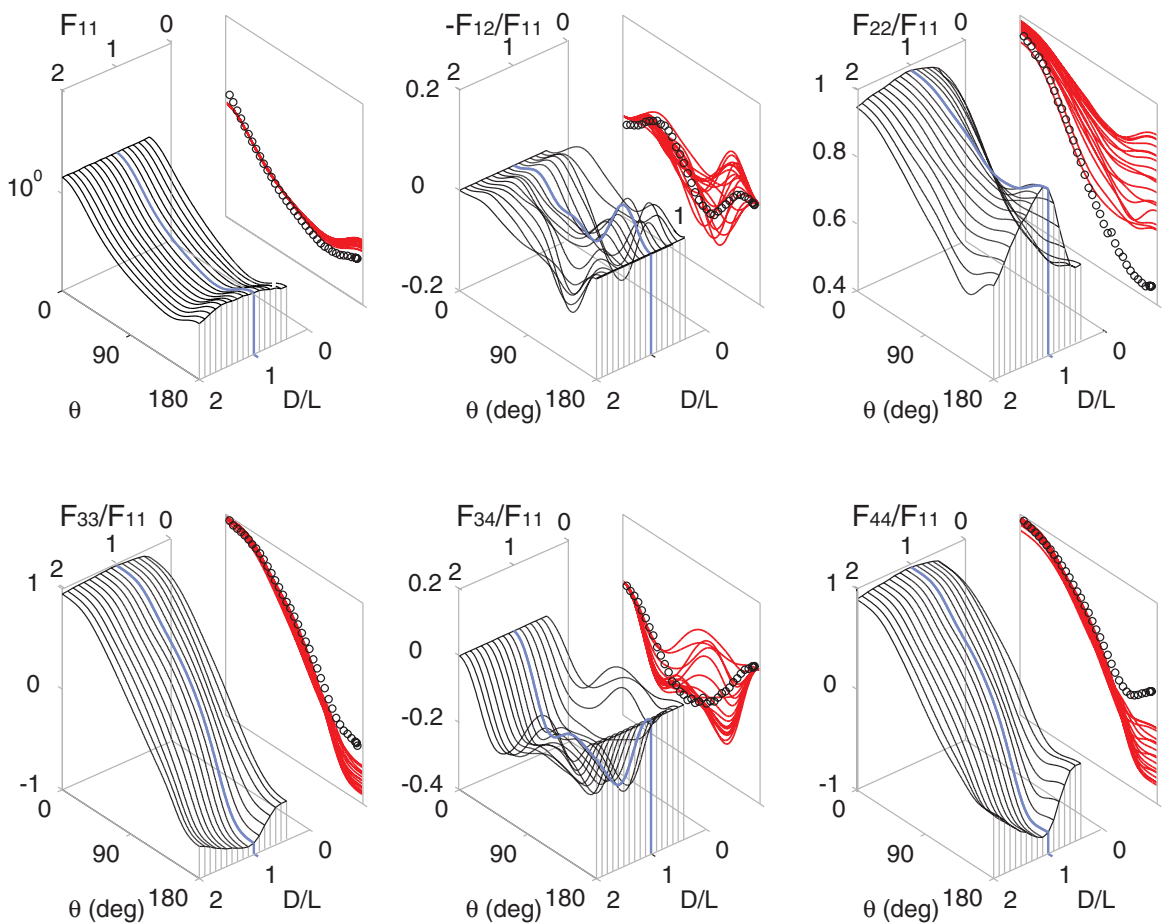

Fig. 10. Same as Fig. 9 but for cylinders whose diameter-to-length ratio are defined by $\mathrm{D} / \mathrm{L}$.

the reflectance is also stronger, the higher $n$ is, except for large angles of incidence. However, these large angles (larger than 70-80 degrees) contribute little to light scattering by randomly oriented particles. In the case of $n=3.0$, it is primarily the real part, $n$, that makes $R$ so large; the role of $k$ is much weaker. Indeed, even for the case $m=3.0+0.0 \mathrm{i}, R$ is significantly larger at each angle of incidence smaller than $\sim 70$ degrees than for $m=1.5+1.0 \mathrm{i}$.

This may help to understand why the scattering behavior of the hematite and rutile samples that have quite large real parts of the refractive index resemble those for volume equivalent spheres. 


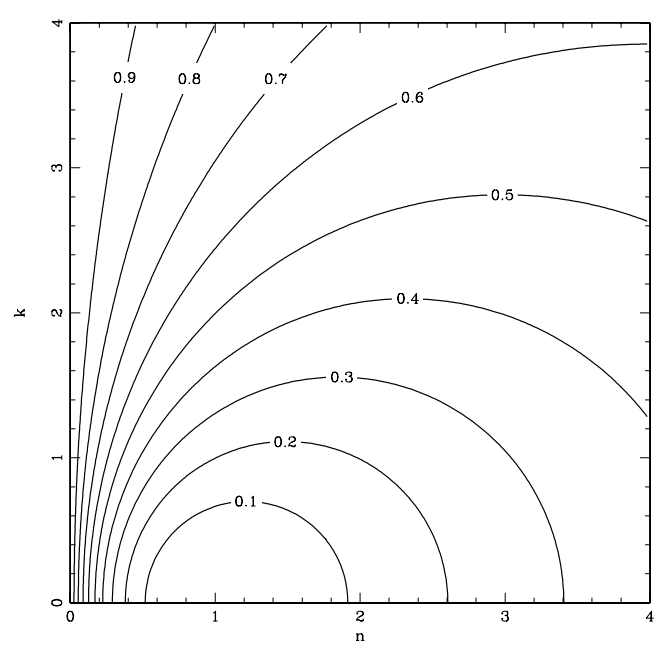

Fig. 11. Reflectance $R$ for normal incidence as a function of the real, $n$, and imaginary, $k$, parts of the refractive index.

\section{Conclusions}

As shown in Sect. 4, the measured scattering patterns for the hematite and rutile samples show remarkable differences when compared with the average scattering matrix for irregular mineral particles with moderate real parts of the refractive indices. The hematite and rutile particles, as well as the samples used to construct the average matrix, consist of particles with shapes with a similar degree of irregularity (see Volten et al. 2001 and Sect. 3 of this paper). The differences in the sizes of the particles of the hematite and rutile samples compared to the sizes of the samples used to obtain the average scattering matrix are more considerable, but previous studies (Muñoz et al. 2000; Volten et al. 2001) and calculations presented in this paper indicate that size differences do not affect the scattering properties as drastically, as is observed for the hematite and rutile particles (see Sect. 4). At the same time, the hematite and rutile samples show a relatively similar scattering behavior despite the large difference in $k$. Therefore, the characteristic scattering behavior presented by the hematite and rutile samples seem to be due to their high real parts of the refractive index. Consequently, we may conclude that for particles with a high value of $n$, the imaginary part of the refractive index plays less of a role.

According to our experimental and theoretical results, the scattering behavior of particles with high real parts of the refractive index does not strongly depend on the shape of the particles. The agreement between the experimentally determined scattering matrix elements as functions of the scattering angle for the irregularly shaped hematite particles and Mie calculations for spheres is reasonably good. This agreement is especially good for the phase function and degree of linear polarization for incident unpolarized light as functions of the scattering angle. Furthermore, we have shown by means of T-matrix calculations for spheroids and cylinders that the scattering matrix as a function of the scattering angle of an ensemble of particles with high real parts is not very dependent on the shape of the particles. Thus, the use of the Mie theory to reproduce the scattering behavior of samples with high real (as well as

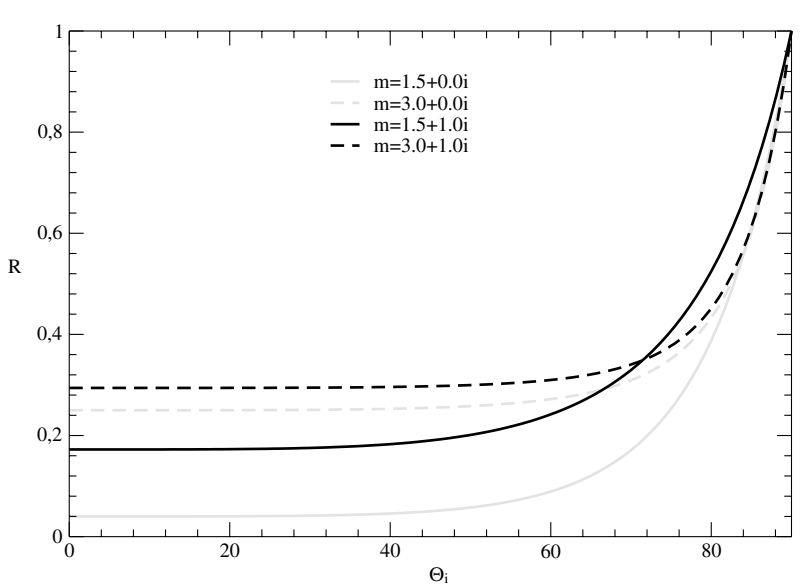

Fig. 12. Reflectance $R$ for oblique incidence as a function of the angle of incidence, $\Theta_{i}$. The calculations were performed for four values of the refractive index as indicated.

imaginary) parts of the refractive index may be considered a reasonably good approach.

Acknowledgements. We are grateful to Michael Mishchenko for fruitful suggestions on a previous version of this manuscript, for providing the second part of the T-matrix code, and for his help with the code. We thank Kemira Pigments Oy for providing the rutile sample. The work of O. Muñoz was partially supported by contracts AYA200403250 and ESP2003-00357.

\section{References}

Banin, A., Ben-Shlomo, T., Margulies, L., et al. 1993, J. Geophys. Res., 98, 20831

Bohren, C. F., \& Huffman, D. R. 1983, Absorption and scattering of light by small particles (New York: John Wiley)

Chan, M. A., Beitler, B., Parry, W. T., Ormo, J., \& Komatsu, G. 2004, Nature, 429, 731

Frondel, J. W. 1975, Lunar Mineralogy (New York: John Wiley)

Hansen, J. E., \& Travis, L. D. 1974, Space Sci. Rev., 16, 527

Höller, R., Ito, K., Tohno, S., \& Kasara, M. 2003, J. Geophys. Res., 108, D23, 8648

Hovenier, J. W. 2000, Measuring scattering matrices of small particles at optical wavelengths, in Light Scattering by Non-Spherical Particles, ed. M. I. Mishchenko, J. W. Hovenier, \& L. D. Travis, Academic, San Diego, 355

Hovenier, J. W., \& van der Mee, C. V. M. 2000, Basic relationships for matrices describing scattering by small particles. In Light Scattering by Non-Spherical Particles, ed. M. I. Mishchenko, J. W. Hovenier, \& L. D. Travis, Academic, San Diego, 61

Hovenier, J. W., Volten, H., Muñoz, O., van der Zande, W. J., \& Waters, L. B. F. M. 2003, J. Quant. Spec. Radiat. Trans., 79-80, 741

Jalava, J.-P., Taavitsainen, V.-M., Lamberg, L., \& Haario, H. 1998, J. Quant. Spec. Radiat. Trans., 60, 399

Jalava, J. P. 2000, Formation of TiO2 Pigment Particles in the Sulphate Process - A Methodological Study, doctor thesis, University of Turku, Finland, ISBN 952-91-2551-8

Jeon, K. S., Winters, J. M., \& Sedlmayr, E. 1999, in Asymptotic Giant Branch Stars, ed. T. Le Bertre, A. Lebre, \& C. Waelkens (San Francisco: ASP), IAU Symp., 191, 233 
Jeon, K. S., Winters, J. M. LeBertre, T. \&, Sedlmayr, E. 2003, A\&A, 407, 191

Konert, M., \& Vandenberghe, J. 1997, Sedimentology, 44, 523

Krotkov, N. A., Flittner, D. E., Krueger, et al. 1999, J. Quant. Spec. Rad. Trans., 63, 613

Mie, G. 1908, Ann. Phys. (Leipzig), 25, 377

Mishchenko, M. I., \& Travis, L. D. 1994, J. Quant. Spec. Radiat. Trans., 51, 759

Mishchenko, M. I., Travis, L. D., \& Mackowski, D. W. 1996, J. Quant. Spec. Radiat. Trans., 55, 535

Mishchenko, M. I., Travis, L. D., Kahn, R. A., \& West, R. A. 1997, J. Geophys. Res., 102, D14, 16831

Mishchenko, M. I., Hovenier, J. W., \& Travis, L. D. 2000a, Light Scattering by Non-Sherical Particles, Academic, San Diego

Mishchenko M. I., Wiscombe, W. J., Hovenier, J. W., \& Travis, L. D. 2000b, Overview of scattering by nonspherical particles, in Light Scattering by Non-Spherical Particles, ed. M. I. Mishchenko, J. W. Hovenier, \& L. D. Travis, Academic, San Diego, 29

Mishchenko, M. I., Travis, L. D., \& Lacis, A. A. 2002, Scattering, Absorption, and Emission of Light by Small Particles (Cambridge University Press)
Morris R. V., \& Lauer, J. V. 1990, J. Geophys. Res., 94, 5105

Muñoz, O., Volten, H., de Haan, J. F., Vassen, W., \& Hovenier, J. W. 2000, A\&A, 360, 777

Muñoz, O., Volten, H., de Haan, J. F., Vassen, W., \& Hovenier, J. W. 2001, J. Geophys. Res., 106, D19, 22833

Muñoz, O., Volten, H. Hovenier, et al. 2004, J. Geophys. Res., 109, D16, D16201

Nousiainen, T., Muinonen, K., \& Raisanen, P. 2003, J. Geophys. Res., 108, 10.1029/2001JD001277

Sokolik, I. N., \& Toon, O. B. 1999, J. Geophys. Res., 104, 9423

Van de Hulst, H. C. 1957, Light Scattering by Small Particles (New York: John Wiley)

Volten, H., Jalava, J. P., de Haan, J. F., Vassen, W., \& Hovenier, J. W. 1999, Appl. Opt., 38, 5232

Volten, H., Muñoz, O., Rol, E., et al. 2001, J. Geophys. Res., 106, 17375

Volten, H., Muñoz, O., Hovenier, J. W., et al. 2005, J. Quant. Spec. Radiat. Trans., 90, 191

West, R. A., Doose, L. R., Eibl, A. M., Tomasko, M. G., \& Mishchenko, M. I. 1997, J. Geophys. Res., 102, 16871

Wriedt, T. 2002, Part. Part. Syst. Charact., 19, 4, 256 